Artículo

\title{
Interacción genotipo ambiente en fracciones de materia seca de trigos harineros sin aristas
}

\author{
Víctor Manuel Zamora Villa ${ }^{\S}$ \\ María Alejandra Torres Tapia \\ Modesto Colín Rico \\ ${ }^{1}$ Departamento de Fitomejoramiento-Universidad Autónoma Agraria Antonio Narro. Calzada Antonio \\ Narro núm. 1923, Col. Buenavista, Saltillo, Coahuila. México. CP. 25315. Tel. 8444110200. \\ (victor.zamora@uaaan.edu.mx; atorres_tapia@hotmail.com; modesto.colin@uaaan.edu.mx). \\ ${ }^{\S}$ Autor para correspondencia: victor.zamora@uaaan.edu.mx.
}

\section{Resumen}

Los cereales representan un importante aporte en la dieta del ganado estabulado durante la época invernal, siendo avena la especie más utilizada, aunque existen otras con potencial forrajero. Se evaluó la producción de forraje seco y sus fracciones en doce genotipos de trigo sin aristas incluyendo tres testigos comerciales de otras especies, con el fin de determinar la magnitud de la interacción genotipo ambiente (IGA), ya que al momento no se cuenta con información al respecto. Se utilizó un diseño de bloques completos al azar con tres repeticiones en cinco ambientes de prueba, sembrando a una densidad de $120 \mathrm{~kg} \mathrm{ha}^{-1}$ y haciendo el manejo tradicional de los cereales de invierno. Las evaluaciones de forraje se realizaron entre los 112 y 118 días después de la siembra. El forraje seco de fracciones se analizó mediante el modelo AMMI. De la IGA detectada en la producción de forraje seco, la mayor cantidad se presentó en los tallos, seguida por la de hojas y finalmente la de espigas. Los genotipos de trigo G6, G1, G11 y G9 se calificaron como deseables junto con la cebada dado que presentaron buen rendimiento y estabilidad. La avena fue la menos productiva y estable. La IGA se manifestó tanto en la producción de forraje seco total como en sus fracciones, siendo los tallos quienes mostraron la mayor interacción. Existen trigos sin aristas que son más deseables que la avena para la producción de forraje y sus fracciones, que pueden ser insertados en esquemas de producción de forraje invernal.

Palabras clave: fracciones de forraje, interacción genotipo ambiente, modelo AMMI, trigo sin aristas.

Recibido: febrero de 2021

Aceptado: abril de 2021 


\section{Introducción}

En algunas áreas del norte de México son importantes los forrajes de corte durante la época invernal porque aportan parte de la dieta del ganado estabulado, ya sea como heno, ensilado o en verde a la fecha la avena continúa siendo la especie más utilizada en invierno, aunque opciones como el triticale, cebada y trigo entre otras especies pudiesen insertarse en los esquemas productivos en tales áreas. Dada la escasez de genotipos de cereales de invierno adecuados para la producción de forraje, es muy importante el desarrollo de nuevas variedades que cubran las necesidades de los productores.

En la formación de nuevos genotipos, la evaluación a través de ambientes es importante para la selección de los mejores genotipos con el fin de: i) utilizarlos como progenitores dentro de algún programa de mejoramiento; y ii) recomendar su uso por los productores en alguna región determinada (Crossa et al., 1990). Para recomendar el uso de un genotipo en una determinada región es importante estimar la estabilidad de producción a través de diferentes ambientes ya que normalmente las variedades evaluadas en ensayos multirregionales se comportan en forma diferencial en los diversos ambientes. Esta respuesta diferencial de los genotipos es llamada interacción genotipo-ambiente (IGA).

Esta ha sido estudiada, descrita e interpretada por medio de varios modelos estadísticos (Crossa, 1990). Diversos modelos han sido usados para estudiar la IGA, uno de ellos fue el propuesto por Eberhart y Russell (1966), que básicamente era una regresión de los rendimientos sobre los índices ambientales, recientemente se ha utilizado el modelo de efectos principales aditivos e interacción multiplicativa (AMMI), por sus siglas en inglés, que ha mostrado ser efectivo en el análisis de ensayos multirregionales o multiambientales, ya que captura una gran proporción de la suma de cuadrados de la IGA, separando en forma precisa los efectos principales de los de interacción (Gauch,1992).

El modelo integra el análisis de varianza y el análisis de componentes principales (Salmerón et al., 1996) y se ha utilizado para evaluar la IGA en cultivos de amplia producción de grano como el trigo (Hristov et al., 2010), cebada (Dyulgerova y Dyulgerov, 2019), arroz (Fasahat et al., 2014) y maíz (López-Morales et al., 2017), sin menospreciar otros muchos trabajos en éstos y otros cultivos.

Desde hace varios años, Ebdon y Gauch (2002) señalaron que la gran mayoría de los trabajos sobre la IGA mediante el modelo AMMI se enfocan en la producción de grano en diversos cultivos y muy pocos evalúan la producción de materia seca (biomasa). En México algunos trabajos con cereales de invierno analizan la producción y estabilidad por cortes sucesivos (Lozano-del Río et $a l ., 2009)$ y no hay alguno que analice la IGA de las fracciones de forraje (hojas, tallos y espigas).

Solo se ha reportado la producción y calidad de estas fracciones en avena (Kilcher y Troelsen, 1973), así como la proporción y calidad del forraje producido por esta especie (Sánchez et al., 2014) y la producción y el valor nutritivo de las fracciones de forraje en trigo (Zamora et al., 2016). Existen reportes de estabilidad de la producción de materia seca en avena (Ahmad et al., 2014) y en veza húngara (Vicia pannonica Crantz) para evaluar la producción de materia seca y semilla (Sayar et al., 2013), mediante la utilización del modelo de Eberhart y Russel (1966). 
En este estudio se analizaron datos de rendimiento de las fracciones de materia seca (hoja, tallos y espigas) con el objetivo de determinar la magnitud de la IGA en doce trigos forrajeros sin aristas y tres testigos de otras especies (avena, cebada y triticale), bajo la hipótesis de que existen trigos harineros sin aristas que poseen baja interacción genotipo ambiente y rendimiento superior a la avena.

\section{Materiales y métodos}

Doce líneas avanzadas de trigo forrajero y las variedades comerciales: Avena $c v$ Cuauhtémoc y el Triticale $c v$ Eronga-83 (Tcl) (Cuadro 1), más una línea experimental de cebada forrajera imberbe (Narro 95) fueron evaluadas durante el ciclo agrícola otoño-invierno (OI) 2010-2011 en el rancho 'Las Vegas' municipio de Francisco I. Madero (A2), en Zaragoza, Coahuila durante los ciclos OI 2010-2011, OI 2015-2016 y OI 2016-2017 (A1, A3 y A5, respectivamente) y San Ignacio, Municipio de San Pedro de las Colonias, Coahuila, ciclo O-I 2016-2017 (A4), mediante un diseño de bloques completos al azar con tres repeticiones.

Cuadro 1. Genotipos evaluados y su identificación.

\begin{tabular}{cccc}
\hline Genotipo & Identificación & Genotipo & Identificación \\
\hline G1 & AN-228-09 & G9 & AN-268-99 \\
G2 & AN-236-99 & G10 & AN-221-09 \\
G3 & AN-244-99 & G11 & AN-326-09 \\
G4 & AN-230-09 & G12 & AN-264-09 \\
G5 & AN-229-09 & G13 & Avena $c v$ Cuauhtémoc \\
G6 & AN-263-99 & G14 & Cebada Narro 95 \\
G7 & AN-217-09 & G15 & Triticale $c v$ Eronga 83 (Tcl) \\
G8 & AN-218-09 & & \\
\hline
\end{tabular}

La combinación particular de una localidad y ciclo de evaluación se denominará genéricamente como ambiente, cuyas características relevantes aparecen en el Cuadro 2.

Cuadro 2. Características y condiciones ambientales de las localidades y ciclos de evaluación.

\begin{tabular}{cccccc}
\hline Localidad y ciclo & Ambiente & $\begin{array}{c}\text { Altitud } \\
(\mathrm{m})\end{array}$ & $\begin{array}{c}\text { Tipo de } \\
\text { suelo }\end{array}$ & $\begin{array}{c}\text { PPacum } \\
(\mathrm{mm})\end{array}$ & T( $\left({ }^{\circ} \mathrm{C}\right)$ max-min \\
\hline Zaragoza OI 2010-11 & A1 & 350 & Calcisol & 22 & $40.3-11$ \\
Fco. I Madero OI 2010-2011 & A2 & 1100 & Regosol & 0 & $38-10$ \\
Zaragoza OI 2015-2016 & A3 & 350 & Calcisol & 134.4 & $34.6-3.3$ \\
San Ignacio OI 2016-2017 & A4 & 1100 & Regosol & 18.2 & $35.3-1.5$ \\
Zaragoza OI 2016-2017 & A5 & 350 & Calcisol & 56 & $39.6-7.3$ \\
\hline
\end{tabular}

PPacum $=$ precipitación acumulada en los meses de evaluación. $\mathrm{T}\left({ }^{\circ} \mathrm{C}\right) \mathrm{Max} ; \mathrm{T}\left({ }^{\circ} \mathrm{C}\right)$; Min= temperaturas máximas y mínimas registradas durante la evaluación. 
La preparación del terreno consistió en las labores tradicionales utilizadas para el establecimiento de cereales de grano pequeño de invierno en las regiones estudiadas bajo condiciones de riego, sembrándose en seco, manualmente a chorrillo, usando una densidad de siembra de $120 \mathrm{~kg} \mathrm{ha}^{-1}$. Se aplicaron 60 unidades de nitrógeno utilizando urea como fuente, más 80 unidades de fósforo utilizando fosfato monoamónico (MAP) para suplir dicho nutriente.

En el primer riego de auxilio se aplicaron 60 unidades adicionales de nitrógeno usando la misma fuente, excepto en San Ignacio, Coahuila donde se aplicaron 100 unidades de nitrógeno en el primer riego de auxilio. Las malezas se controlaron con $1.5 \mathrm{~L} \mathrm{ha}^{-1}$ de 2,4D-Amina y se complementó con deshierbes manuales, pero no se aplicó ningún insecticida o funguicida. A los 118 días después del riego de siembra se realizó un muestreo de forraje en el ciclo OI 2010-2011 en Fco. I Madero y Zaragoza, Coahuila y a los 112 días en el OI 2015-2016 la localidad de Zaragoza, Coahuila, mientras que en el ciclo OI 2016-2017 el muestreo se realizó a los 115 días en San Ignacio y Zaragoza, Coahuila.

La lámina total aplicada durante el ciclo del cultivo fue de $40 \mathrm{~cm}$ aproximadamente en la mayoría de los ambientes, excepto en Zaragoza OI 2015-2016 donde solo se aplicó un riego de auxilio ligero para el llenado de grano, dado que se presentó buena precipitación durante el ciclo de evaluación (Cuadro 2).

La parcela experimental constó de $6.3 \mathrm{~m}^{2}$ (seis hileras de $3 \mathrm{~m}$ de longitud a $0.35 \mathrm{~m}$ entre hileras), muestreando $50 \mathrm{~cm}$ de una de las hileras con competencia completa, cortando a una altura aproximada de $5 \mathrm{~cm}$ sobre la superficie del suelo. Al momento del corte se registraron las variables: altura de planta, rendimiento de forraje verde, etapa fenológica mediante la escala de Zadoks et al. (1974) y el porcentaje de cobertura del terreno, el forraje verde se secó en un asoleadero techado hasta alcanzar peso constante y entonces se determinó la producción de forraje seco total (PSTOT), separando el forraje en sus componentes: hojas (PSH), tallos (PST) y espigas (PSE) para luego convertirlos a t ha ${ }^{-1}$.

La información de la producción de forraje seco y sus fracciones de todos los ambientes de prueba se analizó como bloques completos al azar combinado sobre ambientes para determinar la magnitud y nivel de significancia de la IGA, posteriormente se realizó su análisis mediante modelo AMMI siguiente: $Y_{i j}=\mu+g_{i}+a_{j}+\sum_{k=1}^{n}\left(\lambda_{k} \alpha_{i k} \gamma_{j k}\right)+R_{i j}$. Donde: $Y_{i j}=$ rendimiento del i-ésimo genotipo en el j-ésimo ambiente; $\mu=$ media general; $g_{i}=$ media del $\mathrm{i}$-ésimo genotipo menos la media general; $\mathrm{Aj}=$ media del $\mathrm{j}$-ésimo ambiente menos la media general; $\lambda_{\mathrm{k}}=$ raíz cuadrada del eigenvalor del k-ésimo eje del ACP; $\alpha_{i k}, \gamma_{j k}=$ calificación del ACP para el k-ésimo eje del i-ésimo genotipo y j-ésimo ambiente respectivamente; $\mathrm{R}_{\mathrm{ij}}=$ residual del modelo.

Las calificaciones del análisis de componentes principales (ACP) para los ambientes y genotipos están expresadas como el producto de las unidades del eigenvector correspondiente por la raíz cuadrada del eigenvalor (Zobel et al., 1988). La suma de cuadrados de la interacción genotipoambiente se subdivide en ejes del ACP, donde el eje k posee $\mathrm{g}+\mathrm{a}-1-2 \mathrm{k}$ grados de libertad, donde $\mathrm{g}$ y a representan el número de genotipos y ambientes, respectivamente. Generalmente son retenidos en el modelo sólo los dos primeros componentes principales $(\mathrm{CP})$, los restantes son enviados al residual. 
Las calificaciones asignadas a los genotipos pueden tomar valores positivos o negativos con respecto al componente principal, siendo considerados genotipos estables aquellos que muestren valores de IGA cercanos a cero, valores mayores indicarán una mayor interacción con los ambientes y dependiendo del signo y el cuadrante de la figura generada con los dos primeros $\mathrm{CP}$ se realiza una descripción más detallada de los genotipos, ambientes y su relación. Los análisis se realizaron mediante el programa SAS versión 6.0 (1989).

\section{Resultados y discusión}

Todas las fracciones de forraje evaluadas, así como el forraje seco total mostraron alta significancia en la interacción genotipo ambiente (IGA), justificando así su estudio (Cuadro 3), igual significancia se reportó para los ambientes y genotipos evaluados. El ambiente más productivo fue Zaragoza, Coahuila, OI 2010-2011 (A1) y esa misma localidad, pero en el ciclo OI 2016-2017 (A5) mostró el promedio más bajo de los ambientes evaluados.

Cuadro 3. Cuadrados medios y significancia mediante el modelo AMMI de las variables evaluadas en tres ambientes y media general.

\begin{tabular}{cccccc}
\hline FV & GL & PSTOT & PSH & PST & PSE \\
\hline A & 4 & $510.13^{* *}$ & $45.29^{* *}$ & $176.02^{* *}$ & $24.9^{* *}$ \\
REP & 2 & 13.71 & 2.36 & 1.52 & 0.18 \\
G & 14 & $21.55^{* *}$ & $3.8^{* *}$ & $11.21^{* *}$ & $3.23^{* *}$ \\
IGA & 56 & $7.27^{* *}$ & $1.24^{* *}$ & $2.33^{* *}$ & $0.45^{* *}$ \\
CP1 & 17 & $17.33^{* *}$ & $3^{* *}$ & $5.22^{* *}$ & $0.84^{* *}$ \\
CP2 & 15 & $3.19 \mathrm{~ns}$ & $0.69 \mathrm{~ns}$ & $1.36 \mathrm{~ns}$ & $0.39^{* *}$ \\
\%CP1 & 17 & 72.38 & 73.58 & 68 & 56.51 \\
\%CP2 & 15 & 11.76 & 15.03 & 15.65 & 23.25 \\
EE & 148 & 4.19 & 0.69 & 1.39 & 0.18 \\
Media & & 11.05 & 4 & 5.66 & 1.39 \\
CV & & 18.8 & 20.8 & 20.8 & 30.6 \\
\hline
\end{tabular}

$\mathrm{FV}=$ fuente de variación; $\mathrm{GL}=$ grados de libertad; $\mathrm{PSTOT}=$ peso seco total; $\mathrm{PST}=$ peso seco de tallos, $\mathrm{PSH}=$ peso seco de hojas; PSE= peso seco de espigas; $\mathrm{A}=$ ambiente; $\mathrm{G}=$ genotipo; $\mathrm{IGA}=$ interacción genotipo ambiente; $\mathrm{CP} 1$ у CP2= componentes principales 1 y $2 ; \% \mathrm{CP} 1$ y $\% \mathrm{CP} 2=$ porcentaje de varianza explicada por los componentes principales 1 y 2 , respectivamente; $\mathrm{EE}=$ error experimental; $\mathrm{CV}=$ coeficiente de variación.

El genotipo más productivo para PSTOT fue la cebada Narro95 (G14) seguida por los genotipos de trigo sin aristas: G6 (AN-263-99), G1 (AN-228-09), G11 (AN-326-09) y G9 (AN-268-99) con más de $11 \mathrm{t} \mathrm{ha}^{-1}$ de materia seca, en tanto que el menos productivo fue la avena $c v$ Cuauhtémoc con $8.3 \mathrm{t} \mathrm{ha}^{-1}$. Torres et al. (2019) han reportado un rendimiento promedio de $7.9 \mathrm{t} \mathrm{ha}^{-1}$ para esta misma variedad de avena al evaluarla en dos localidades de Coahuila. Esto permite afirmar la existencia de trigos con potencial productivo superior a dicho testigo comercial.

El análisis AMMI explicó al menos 79.76\% de la IGA en el forraje seco de espigas (PSE), siendo la fracción en la cual se obtuvo la menor explicación; en tanto que el forraje seco total (PSTOT), forraje seco de hojas y tallos (PSH y PST, respectivamente) fueron explicados en más de $80 \%$ de su IGA con los dos primeros CP. 
En estas últimas variables, solo el primer componente principal resultó altamente significativo, mientras que para PSE los dos primeros componentes resultaron altamente significativos, sugiriendo que las interacciones de los genotipos con los ambientes fueron más complejas en PSE que las detectadas en las otras fracciones, debido probablemente a diferencias en precocidad de los cereales estudiados y que sin duda influyó en el coeficiente de variación reportado en dicha variable, tal como se aprecia en el (Cuadro 3).

La mayor varianza de IGA se presentó en el PSTOT seguido por el PST, PSH y finalmente el PSE. De los pocos trabajos sobre estabilidad de la producción de forraje con cereales de invierno, Lozano-del Río et al. (2009) han reportado la existencia de IGA y al analizarla mediante el modelo AMMI les permitió calificar los grupos de genotipos estudiados y clasificarlos de acuerdo con dicha metodología, aunque ellos tuvieron una menor explicación de la IGA en comparación con la aquí reportada.

Las fracciones que más contribuyeron al PSTOT fueron los tallos $(50.5 \%)$, seguidos por las hojas (37.3\%) y finalmente las espigas (12.2\%), coincidiendo con lo reportado para estos cereales respecto a la contribución de sus fracciones (Zamora et al., 2016). Al realizar la gráfica con los dos componentes principales (CP) del análisis AMMI para PSTOT, se aprecia que los genotipos más estables fueron el G1, G4 y el G3 (AN-228-09, AN-230-09 y AN-244-99, respectivamente), los cuales se ubicaron cerca del cruce de las líneas que parten del punto cero de ambos componentes principales (cero IGA) de la Figura 1.

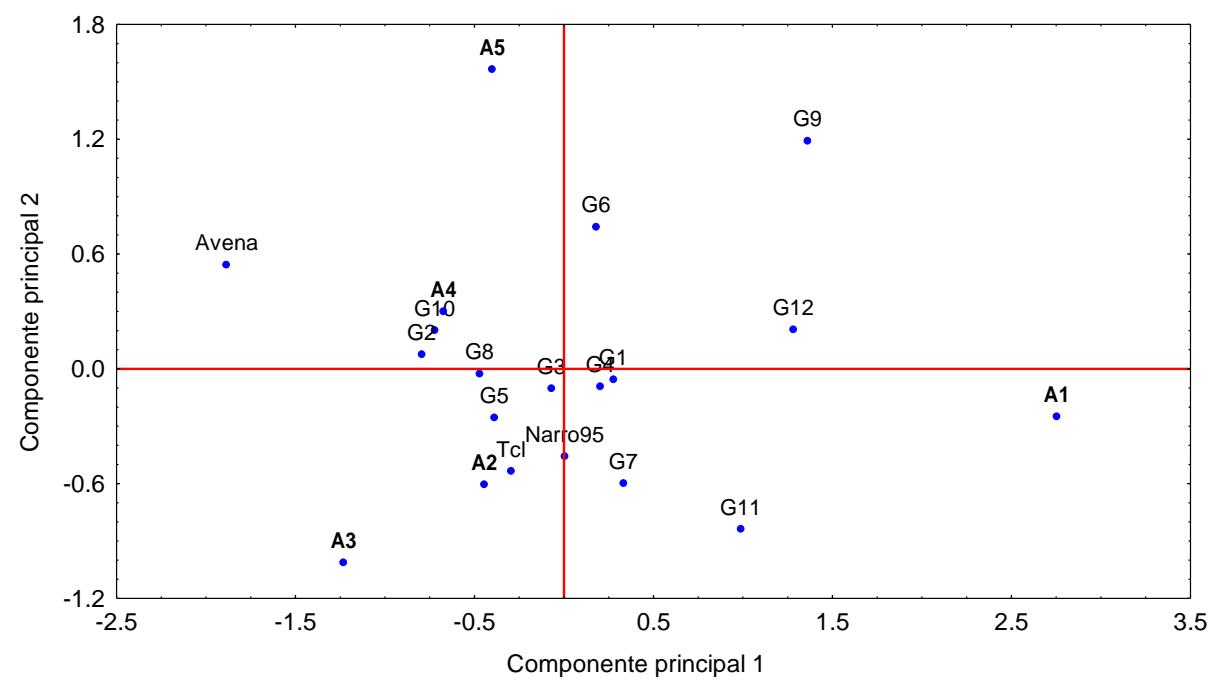

Figura 1. Interacción genotipo ambiente para forraje seco total en los genotipos evaluados (G) y ambientes (A) de prueba.

El genotipo 3 (AN-244-99) presentó interacciones pequeñas negativas con ambos componentes. Las mayores interacciones positivas (con ambos CP) las presentó el genotipo 9 de trigo (AN-26899), de tal forma que debido a sus interacciones positivas puede producir un poco más de la media expresada, algo similar ocurrió con los genotipos G6 y G12 (AN-263-99 y AN-264-99), mientras que la avena $c v$ Cuauhtémoc presentó interacciones negativas con el primer componente y positivas con el segundo CP, lo que se espera menor producción de la expresada como promedio (Figura 1). 
El A3 (Zaragoza OI 2015-2016), fue el ambiente que provocó las interacciones más negativas en la producción de forraje posiblemente debido a las condiciones de alta precipitación registradas en ese ciclo. En Zaragoza OI 2010-11 (A1) se asociaron los genotipos AN-217-09 y AN-326-09 (G7 y G11) indicando que fue en este ambiente donde mejor produjeron, de igual forma en los ambientes Fco. I Madero OI 2010-2011 (A2) y Zaragoza OI 2015-2016 (A3), los genotipos AN229-09 (G5), la cebada Narro 95 y el triticale $c v$ Eronga 83 (Tcl) fueron los que mejores producciones de forraje seco total alcanzaron. De manera similar se establece la asociación entre ambientes y genotipos en los demás cuadrantes de dicha figura.

Al graficar los genotipos y ambientes en el plano generado por el rendimiento del forraje seco total y el primer componente del AMMI (Figura 2), se puede calificar a los genotipos: cebada Narro95 y trigos G6 (AN-263-99), G1 (AN-228-09), G11 (AN-326-09) y G9 (AN-268-99) como genotipos deseables, ya que mostraron los mayores rendimientos con respecto a la media general (línea punteada) e interacciones pequeñas y positivas (distancia con respecto a la línea sólida). Los genotipos AN-244-99 (G3) y AN-229-09 (G5) superaron también la media general, pero exhibieron interacciones pequeñas y negativas. La avena fue la menos rendidora de los genotipos y mostró interacciones grandes y negativas.

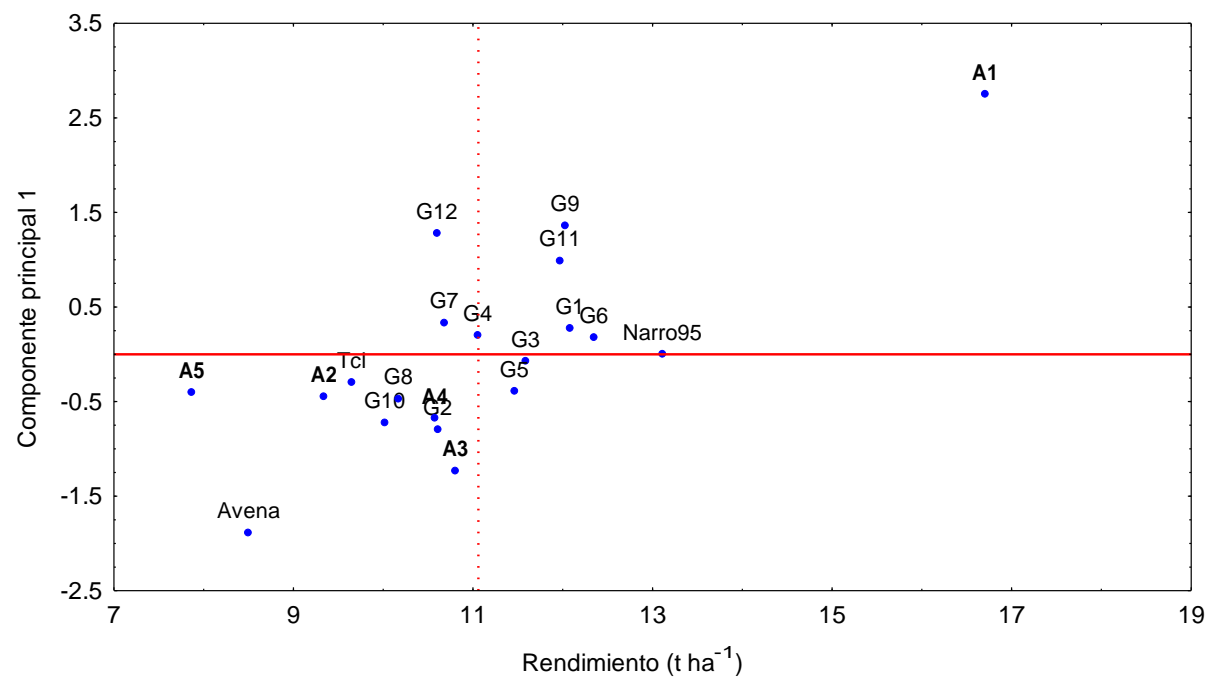

Figura 2. Genotipos (G) y ambientes (A) con base en el rendimiento de forraje seco total y primer CP del análisis AMMI.

Esto sugiere que la avena fue muy afectada por el ambiente y que existen genotipos más productivos y de mayor estabilidad de su producción de forraje seco total, como los mencionados al inicio de este párrafo. Recientemente, Torres et al. (2019) han reportado la existencia de cebadas forrajeras que superan el rendimiento de la avena $c v$ Cuauhtémoc al evaluarlas en dos localidades de Coahuila. Se enfatiza la superioridad de los genotipos de trigo sobre la avena $c v$ Cuauhtémoc, ya que es una de las variedades que más se utilizan en la producción de forraje invernal, donde el estado de Coahuila se ubica en el quinto lugar por su volumen de producción (SIAP, 2015).

De los ambientes evaluados A1 (Zaragoza OI 2010-2011) fue el de mayor promedio y provocó interacciones grandes y positivas, mientras el ambiente más desfavorable para la producción de forraje seco total fue el A5 (Zaragoza OI 2016-2017) y provocó pequeñas interacciones negativas, 
los ambientes A2 y A4 se ubicaron por debajo de la media y provocaron pequeñas interacciones negativas, tal como se aprecia en la Figura 2. El A3 provocó las interacciones más negativas, tal como se asentó al discutir la Figura 1.

Al analizar la IGA para producción de tallos, se calificó a los genotipos AN-228-09, AN-230-09, AN-244-99 y AN-218-09 (G1, G4, G3 y G8, respectivamente) como los más estables, ya que se ubicaron cerca del cruce de las líneas que marcan el punto cero de ambos componentes, sugiriendo interacciones pequeñas y positivas para AN-228-09 (G1), mientras que genotipos como el G3 (AN244-99) y el G8 (AN-218-09) presentaron interacciones negativas de poca magnitud, tal como se aprecia en la Figura 3.

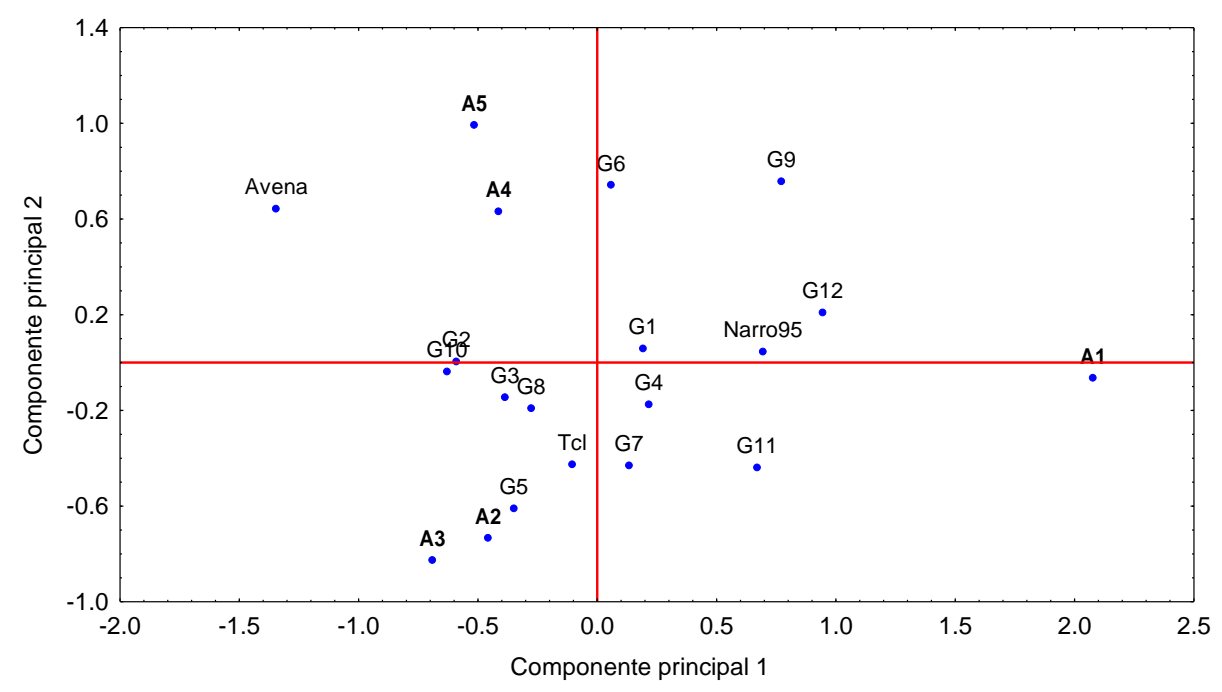

Figura 3. Interacción genotipo ambiente para producción de forraje seco de tallos en los genotipos (G) y ambientes (A) evaluados.

Los genotipos G9 (AN-268-99) y G12 (AN-264.09) presentaron las interacciones más grandes y positivas que las exhibidas por la cebada Narro95. La avena $c v$ Cuauhtémoc por su parte mostró grandes interacciones negativas con el $\mathrm{CP} 1$ y positivas con el $\mathrm{CP} 2$, asociándose mayormente a los ambientes A4 y A5 en los cuales produjo mayor forraje seco de tallos. De los ambientes, A3 y A2 provocaron las mayores interacciones negativas para esta variable.

Los efectos ambientales continuaron de manera similar que cuando se analizó la producción de forraje seco total dado que la fracción de tallos contribuyó en mayor porcentaje $(50.5 \%$, tal como se mencionó anteriormente).

Se calificaron como genotipos deseables para la producción de forraje seco de tallos a G1 (AN228-09) y G6 (AN-263-99), seguidos por la cebada Narro95, (G9) (AN-268-99) y G11 (AN-32609) (Figura 4), quienes mostraron interacciones pequeñas y positivas. La avena $c v$ Cuauhtémoc se manifestó nuevamente como la de menor rendimiento de forraje seco de tallos y grandes interacciones negativas. El ambiente A1 continuó siendo el más favorable y A5 el menos favorable para la producción de materia seca de tallos, en tanto que A3 mostró las interacciones más negativas. 


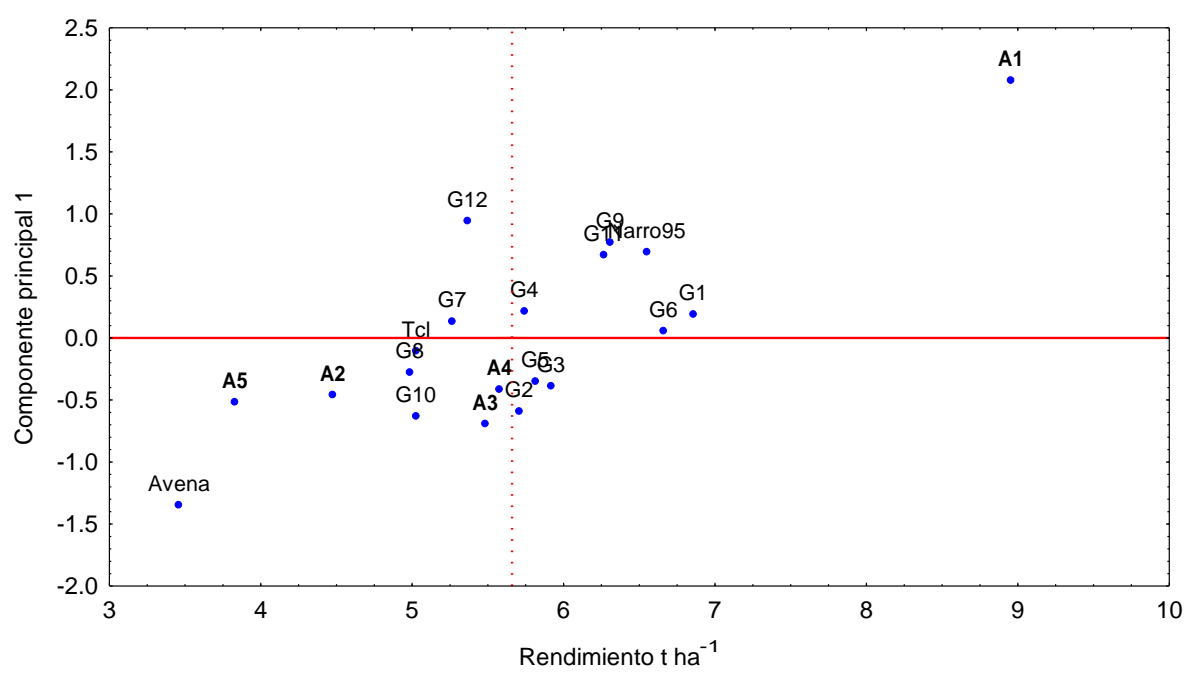

Figura 4. Genotipos (G) y ambientes (A) con base en el rendimiento de forraje seco de tallos y el primer CP del análisis AMMI.

Dado que la principal fracción de forraje en estos genotipos fueron los tallos, no es de extrañarse que el comportamiento del PSTOT y PST sean similares en su IGA. Feyissa et al. (2008), en un estudio con 20 variedades de avena concluyeron que aquellas variedades con mayor proporción de tallos podrían ser útiles para el ensilaje, por lo que los genotipos antes mencionados podrían recomendarse para tal fin.

Para el forraje seco de hojas, el análisis de la IGA calificó a los genotipos G6, G4, G10 y G1 (AN263-99, AN-230-09, AN-221-09 y AN-228-09, respectivamente) como los de menor interacción, ya que se encuentran ubicados cerca del cruce de las líneas que marcan el punto cero de ambos componentes. Genotipos como la avena $c v$ Cuauhtémoc y cebada Narro95 presentaron las mayores interacciones negativas y se asociaron bien a los ambientes A3 y A5 que provocaron las mayores interacciones negativas para esta variable (Figura 5).

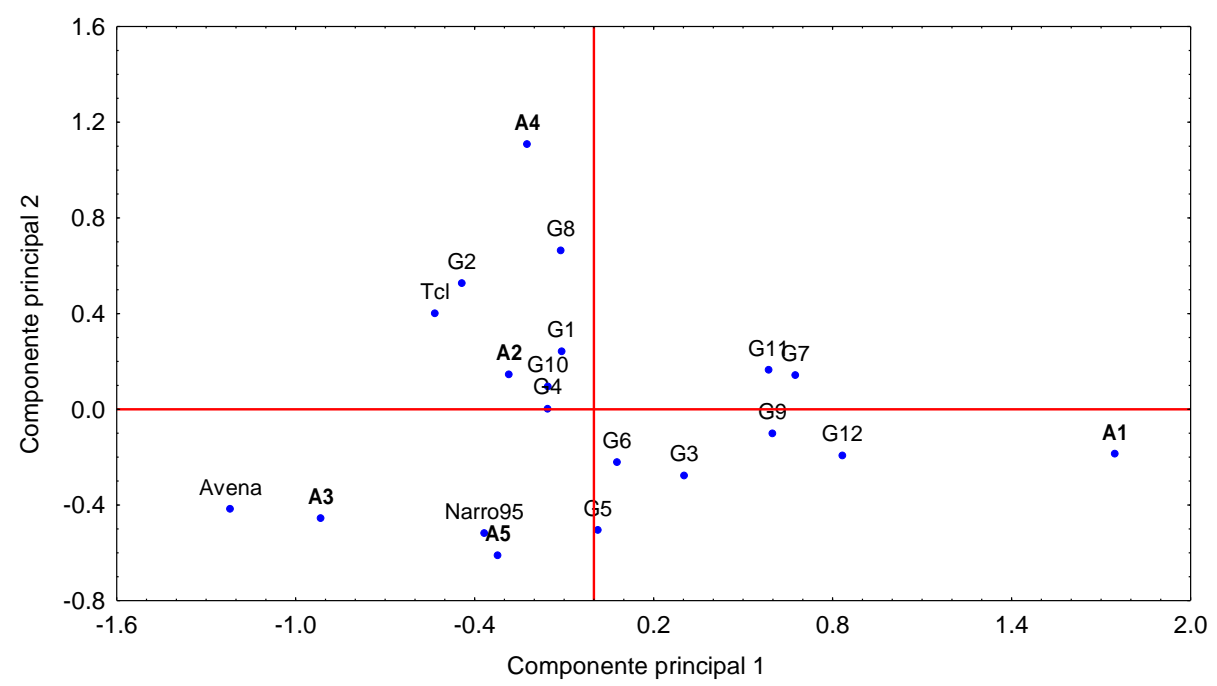

Figura 5. Interacción genotipo ambiente para forraje seco de hojas en los genotipos (G) y ambientes (A) evaluados. 
Aunque la avena $c v$ Cuauhtémoc y la cebada Narro95 mostraron la mayor producción de forraje seco de hojas, no necesariamente fueron las más deseables para esta variable, ya que ambas mostraron interacciones negativas, siendo mayores en la avena $c v$ Cuauhtémoc (Figura 6), sugiriendo que su producción de hojas es fuertemente afectada por las condiciones ambientales donde se desarrollan.

Los trigos AN-244-99 y AN-217-09 (G3 y G7, respectivamente) con rendimientos similares al de avena $c v$ Cuauhtémoc y cebada Narro 95, mostraron interacciones positivas, sugiriendo que pueden aprovechar las condiciones favorables para producir mayor cantidad de hojas. Para esta variable, la menor cantidad de hojas las produjo el triticale $c v$ Eronga $83(\mathrm{Tcl})$ quien mostró también interacciones negativas. De nuevo el A3 provocó las interacciones más negativas y el A1 las positivas.

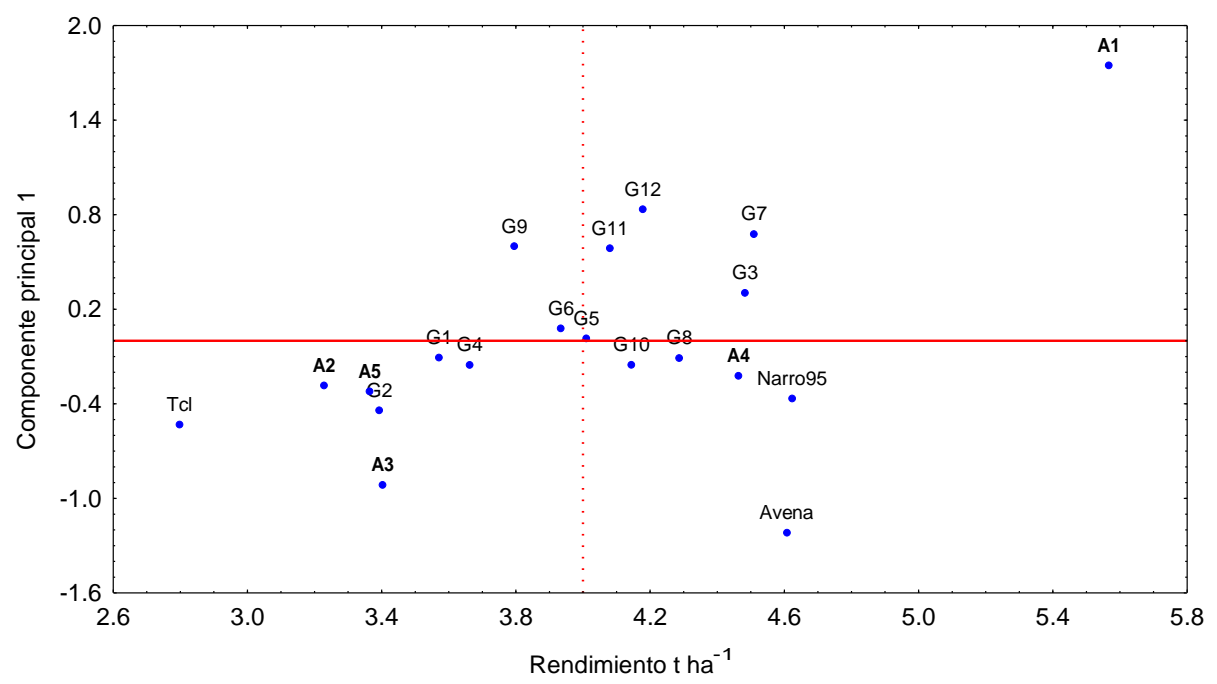

Figura 6. Genotipos (G) y ambientes (A) con base en el rendimiento de forraje seco de hojas y el primer CP del análisis AMMI.

De acuerdo con Feyissa et al. (2008), los genotipos con mayor proporción de hojas en un determinado estado de crecimiento podrían recomendarse para la henificación, por lo que genotipos como los enlistados arriba pudieran recomendarse para este tipo de conservación de forraje.

Para la producción de forraje seco de espigas, el genotipo G12 (AN-264-09) se comportó como el de menor IGA con pequeñas interacciones negativas, en tanto genotipos como el G9 (AN-268-99) y G1 (AN-228-09) presentaron las mayores interacciones positivas. La cebada Narro 95 presentó las interacciones más negativas con el primer componente y positivas con el segundo, asociándose positivamente con los ambientes A3 y A4 donde produjo la mayor cantidad de espigas, tal como se aprecia en la Figura 7.

La avena $c v$ Cuauhtémoc junto con los trigos AN-244-99, AN-229-09, AN-217-09, AN-218-09 y AN-221-09 (genotipos G3, G5, G7, G8, y G10, respectivamente) mostraron interacciones negativas y se asociaron al A2, en tanto que el A1 continuó siendo el ambiente que provocó interacciones positivas. 


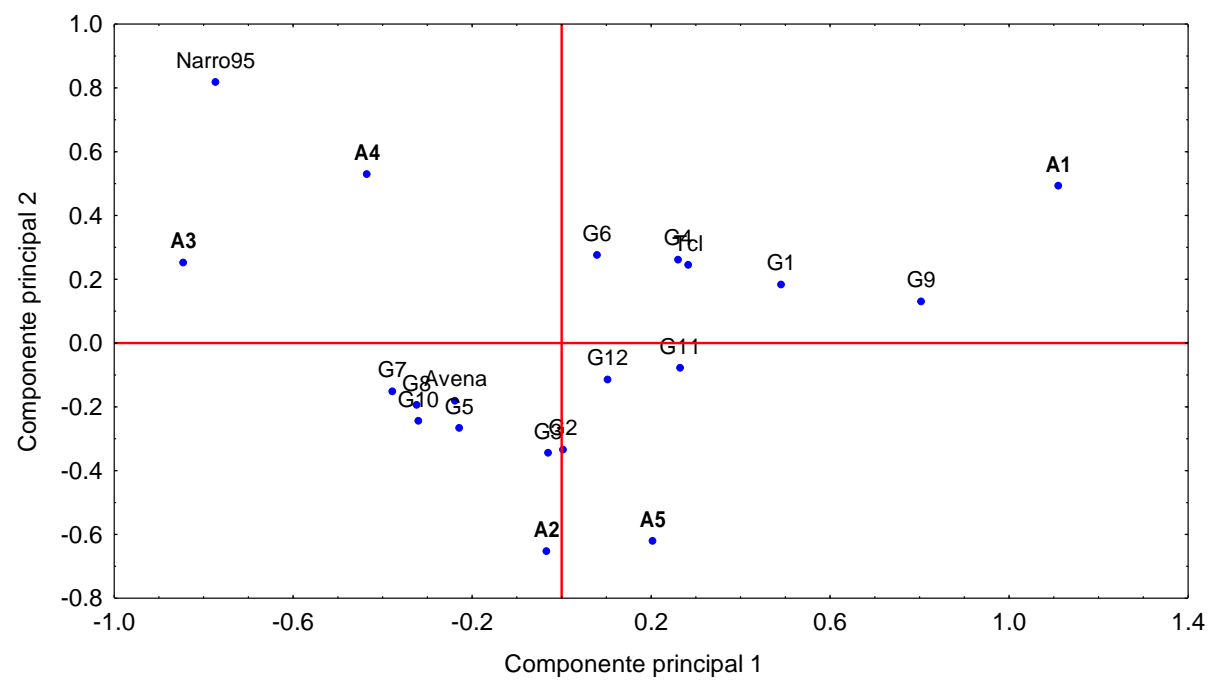

Figura 7. Interacción genotipo ambiente para producción de forraje seco de espigas en los genotipos (G) y ambientes (A) evaluados.

La Figura 8 permite apreciar que el genotipo G9 de trigo sin aristas (AN-268-99) y la cebada Narro 95 produjeron la mayor cantidad de peso seco de espigas, pero la cebada exhibió grandes interacciones negativas mientras AN-268-99 (G9) las exhibió grandes y positivas, resultando deseable un genotipo como este trigo ya que tiene una producción similar a la cebada pero puede aprovechar las condiciones ambientales para incrementar la cantidad de forraje seco de espigas, comportamiento que exhiben también el triticale $c v$ Eronga 83 (Tcl) y AN-263-99 (G6).

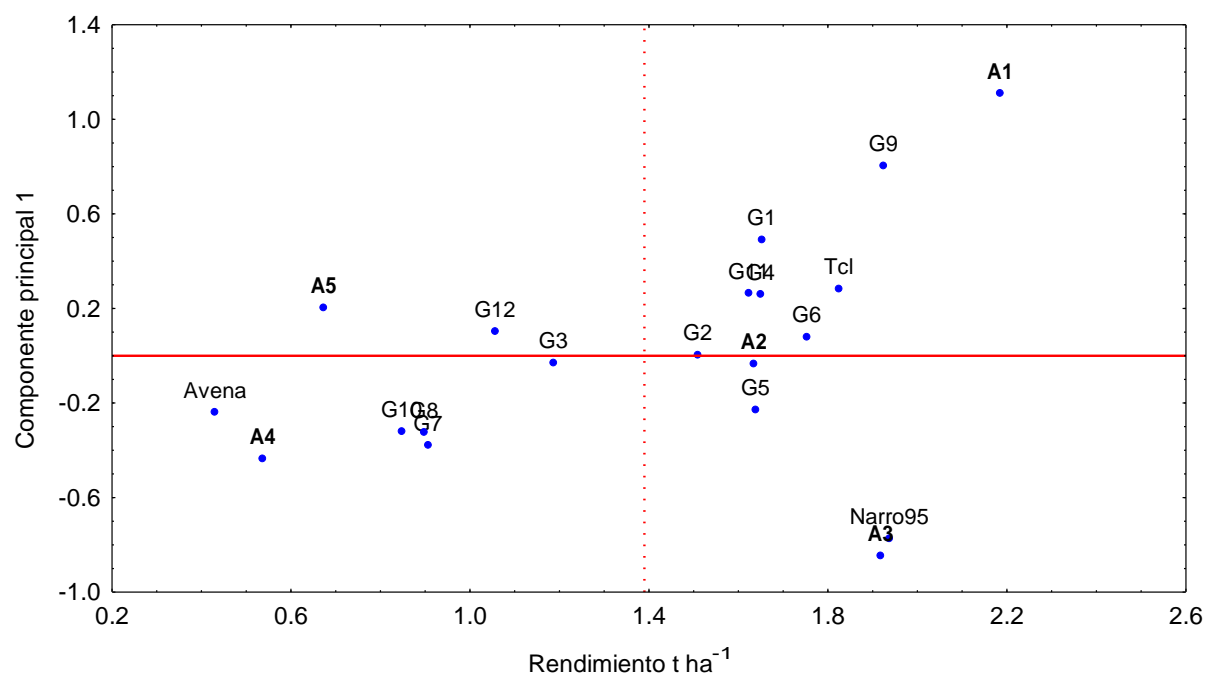

Figura 8. Genotipos (G) y ambientes (A) con base en el rendimiento de forraje seco de espigas y el primer CP del análisis AMMI.

En el extremo opuesto aparece la avena $c v$ Cuauhtémoc mostrando los menores rendimientos de esta fracción de forraje e interacciones negativas. Para esta variable el ambiente A2 provocó las interacciones más negativas y A1 continuó apareciendo como el ambiente más favorable para la producción de forraje seco y sus fracciones de los cereales estudiados. 
Es en la producción de esta fracción de forraje donde cobra importancia la utilización de genotipos sin aristas, ya que la presencia de éstas en etapas avanzadas del o los cereales que la poseen, pueden lacerar las mucosas de los animales que los consuman.

\section{Conclusiones}

Con lo antes asentado se puede concluir que la IGA se presentó tanto en el forraje seco total como en las fracciones que lo componen, y la fracción de forraje que presentó mayor IGA fue la correspondiente a los tallos, seguida por las hojas y en menor cuantía la de espigas. En este estudio la avena $c v$ Cuauhtémoc fue el genotipo que mostró las mayores interacciones negativas en el rendimiento de forraje y sus fracciones, así como menor producción, mientras que la mayor producción se obtuvo con la cebada Narro 95.

Existen trigos sin aristas como el AN-228-09 (G1), AN-263-99 (G6) y AN-268-99 (G5) que se desempeñaron mejor que la avena en la producción de forraje seco y sus fracciones, exhibiendo menor IGA por lo que pueden ser utilizados en esquemas de producción de forraje en la temporada invernal.

\section{Agradecimientos}

Los autores agradecen a la Universidad Autónoma Agraria Antonio Narro por todas las facilidades y apoyos brindados para la realización de este trabajo.

\section{Literatura citada}

Ahmad, M.; Zaffar, G.; Dar, Z. A.; Saleem, N. and Habib, M. 2014. Parametric stability analyses for green forage yielding traits in oats (Avena sativa, L.). Afr. J. Agric. Res. 9(11):10081011. Doi:10.5897/AJAR2013.7860.

Crossa, J. 1990. Statistical analysis of multilocation trials. USA. Adv. Agron. 44(1):55-85.

Crossa, J. L.; Gauch, H. G. and Zobel, R. W. 1990. Additive main effects and multiplicative interaction analysis of two international maize cultivar trials. USA. Crop Sci. 30(3):493-500.

Dyulgerova, B. and Dyulgerov, N. 2019. Genotype by environment interaction for grain yield of barley mutant lines. Agriculture (Pol'nohospodástvo). 65(2):51-58.

Ebdon, J. S. and Gauch, H. G. 2002. Additive main effect and multiplicative interaction analysis of national turfgrass performance trial: I. Interpretation of genotype $\mathrm{x}$ environment interaction. USA. Crop Sci. 42(2):489-496.

Eberhart, S. A. and Russell, W. A. 1966. Stability parameters for comparing varieties. USA. Crop Sci. 6(1):36-40.

Fasahat, P.; Muhammad, K.; Abdullah, A.; Rahman, B. M. A.; Gauch, J. H. G. and Ratnam, W. 2014. Genotype environment assessment for grain quality traits in rice. Comm. Biom. Crop Sci. 9(2):71-82.

Feyissa, F.; Tolera, A. and Melaku, S. 2008. Proportions of morphological fractions of oats (Avena sativa, L.) as affected by variety and growth stage. Livestock Res. Rural Development. 20(6):artícle89. http://www.lrrd.org//rrd20/6/feyi20089.htm.

Gauch, H. G. 1992. Statistical analysis of regional yield trials: AMMI analysis of factorial designs. Elsevier, Amsterdam. The Netherlands. 278 p. 
Hristov, N.; Mladenov, N.; Djuric, V.; Kondic, S. A.; Marjanovic, J. A. and Simic, D. 2010. Genotype by environment interactions in wheat quality breeding programs in southeast Europe. Euphytica. 174(3):315-324.

Kilcher, M. R. and Troelsen, J. E. 1973. Contribution and nutritive value of the major plant components of oats through progressive stages of development. Can. J. Plant Sci. 53(2):251-256.

López-Morales, F.; Vázquez-Carrillo M. G.; Molina-Galán, J. D.; García-Zavala, J. J.; CoronaTorres, T.; Cruz-Izquierdo, S.; López-Romero, G.; Reyes-López, D. y Esquivel-Esquivel, G. 2017. Interacción genotipo-ambiente, estabilidad del rendimiento y calidad de grano en maíz Tuxpeño. Rev. Mex. Cienc. Agríc. 8(5):1035-1050.

Lozano-del Río, A. J.; Zamora-Villa, V. M.; Ibarra-Jiménez, L.; Rodríguez-Herrera, S. A.; CruzLázaro E. y Rosa-Ibarra, M. 2009. Análisis de la interacción genotipo-ambiente mediante el modelo AMMI y potencial de producción de triticales forrajeros (x Triticosecale, Wittm,). Universidad y Ciencia. 25(1):81-92.

Salmerón, Z. J. J.; Cabañas, B. C.; Chávez, J.; y Valenzuela. M. V. 1996. Agrupación de ambientes de temporal y genotipos de avena con el modelo AMMI. Rev. Fitotec. Mex. 19(2):151-162.

Sánchez, G. R. A.; Gutiérrez, B. H.; Serna, P. A.; Gutiérrez, L. R. y Espinoza, C. A. 2014. Producción y calidad de forraje de variedades de avena en condiciones de temporal en Zacatecas, México. Rev. Mex. Cienc. Pec. 5(2):131-142.

SAS Institute Inc. 1989. SAS/STAT User's guide. Versión 6. (Ed.). SAS Institute Inc., Cary, NC. $943 \mathrm{p}$.

Sayar, M. S.; Anlarsal, A. E. and Basbag, M. 2013. Genotype-environment interactions and stability analysis for dry-matter yield and seed yield in hungarian vetch (Vicia pannonica CRANTZ.). Turkish J. Field Crops. 18(2):238-246.

SIAP. 2015. Atlas agroalimentario 2015. Primera edición. Sistema de Información Agroalimentaria y Pesquera. Secretaría de Agricultura, Ganadería, Desarrollo Rural, Pesca y Alimentación México. 216 p.

Torres, T. M. A.; Zamora, V. V. M.; Colín, R. M.; Foroughbakch, P. R. y Ngangyo, H. M. 2019. Caracterización y agrupamiento de cebadas imberbes mediante sensores infrarrojos y rendimiento de forraje. Rev. Mex. Cienc. Agríc. 10(5):1125-1137.

Zadoks, J. C.; Chang, T. T. and Konzak. C. F. 1974. A decimal code for the growth stages of cereals. Eucarpia Bulletin. 7(1):42-52.

Zamora, V. V. M.; Colín, R. M.; Torres, T. M. A.; Rodríguez, G. A. y Jaramillo S. M. A. 2016. Producción y valor nutritivo en fracciones de forraje de trigos imberbes. Rev. Mex. Cienc. Agríc. 7(2):291-300.

Zobel, R. W.; Wright, M. J. and Gauch, H. G. 1988. Statistical analysis of a yield trial. Agron. J. 80(3):388-393. 